\title{
Pediatric granular cell tumor in the posterior wall of the larynx extending to the trachea
}

\author{
Jungsuk Ahn ${ }^{1}$, Na Rae Kim ${ }^{1}$, Yong Han Sun ${ }^{2}$ \\ Departments of ${ }^{1}$ Pathology and ${ }^{2}$ Pediatrics, Gil Medical Center, Gachon University College of Medicine, Incheon, Korea
}

\begin{abstract}
Granular cell tumor (GCT) is a slow-growing benign neoplasm that can be found in any organ. Pediatric laryngotracheal GCT is rare. We experienced a 6-year-old boy suffering from a barking cough and symptoms of stridor and croup for one month. Head and neck computed tomography revealed a protruding mass that occluded $60 \%$ of the airway lumen. Under the impression of hemangioma or papilloma, excision revealed a submucosal non-encapsulated mass. Histologically, the mass was composed of sheets of large polyhedralshaped tumor cells containing plump eosinophilic granular cytoplasm and centrally placed, small, bland-appearing nuclei. The tumor cells were positive for S-100 protein, and voluminous eosinophilic cytoplasm was stained by diastase-resistant periodic acid-Schiff. The present report describes a unique case of a huge pediatric laryngeal GCT extending to the subglottic trachea. We also review the clinical course of pediatric laryngotracheal GCT and emphasize the importance of diagnosing GCT in children.
\end{abstract}

Key Words: Granular cell tumor; Trachea; Larynx; Children

Received: December 10, 2019 Revised: February 3, 2020 Accepted: February 27, 2020

Corresponding Author: Na Rae Kim, MD, Department of Pathology, Gil Medical Center, Gachon University College of Medicine, 21 Namdong-daero 774beon-gil, Namdong-gu, Incheon 21565, Korea

Tel: +82-32-460-3073, Fax: +82-32-460-2394, E-mail: clara_nrk@gilhospital.com

Granular cell tumor (GCT) has been variably called granular cell myoblastoma, Abrikossoff's tumor, and granular cell schwannoma [1]. As its name indicates, GCT is regarded to be of neural crest Schwann cell origin, like schwannoma, although its pathogenesis is still under debate. Its characteristic granularity is caused by accumulated secondary lysosomes in the cytoplasm of the tumor cells [1]. Recently, we experienced a six-year-old boy with a huge GCT in the larynx that extended to the trachea. Here, we describe a pediatric patient with laryngotracheal GCT who was difficult to diagnose early.

\section{CASE REPORT}

A previously healthy 6-year-old boy presented with acute onset noisy breathing, hoarse voice, and symptoms of croup and stridor for three weeks. He had a history of asthma for the previous 1 year, and there was no family history. Physical examination was nonspecific. Laryngoscopy revealed posterior vocal cord swelling and a crust-like lump lesion (Fig. 1A). Laryngitis or tracheitis was suspected. Head and neck computed tomography
(CT) showed a $22 \times 9$-mm-sized lesion with a lobulated margin and heterogeneous enhancement in the posterior wall of the subglottic trachea, suspicious for papilloma or hemangioma (Fig. 1B). In addition, the mass arose from the cricoid cartilage at the posterior laryngeal wall and extended to the subglottic trachea level. With the patient under laryngeal mask airway general anesthesia, suspension laryngoscope setting bagging was performed using a $4.5-\mathrm{mm}$ endotracheal tube. The posteriorly located glottic mass was incompletely removed using a cup forceps, and the mucosal surfaces of both sides of the mass were left intact to prevent stenosis of the airway.

Histologically, the excised mass from the trachea and glottis was composed of large polyhedral cells arranged in sheets with plump eosinophilic granular cytoplasm (Fig. 2A). Centrally placed small nuclei were bland with or without nucleoli. Mitosis and necrosis were absent. The overlying epithelium was observed as either intact or as occasional erosive squamous epithelium with focal pseudoepitheliomatous hyperplasia. The eosinophilic granular cytoplasm was stained with diastase-resistant periodic acid-Schiff (PAS) (Fig. 2B, left). The polyhedral large cells were 

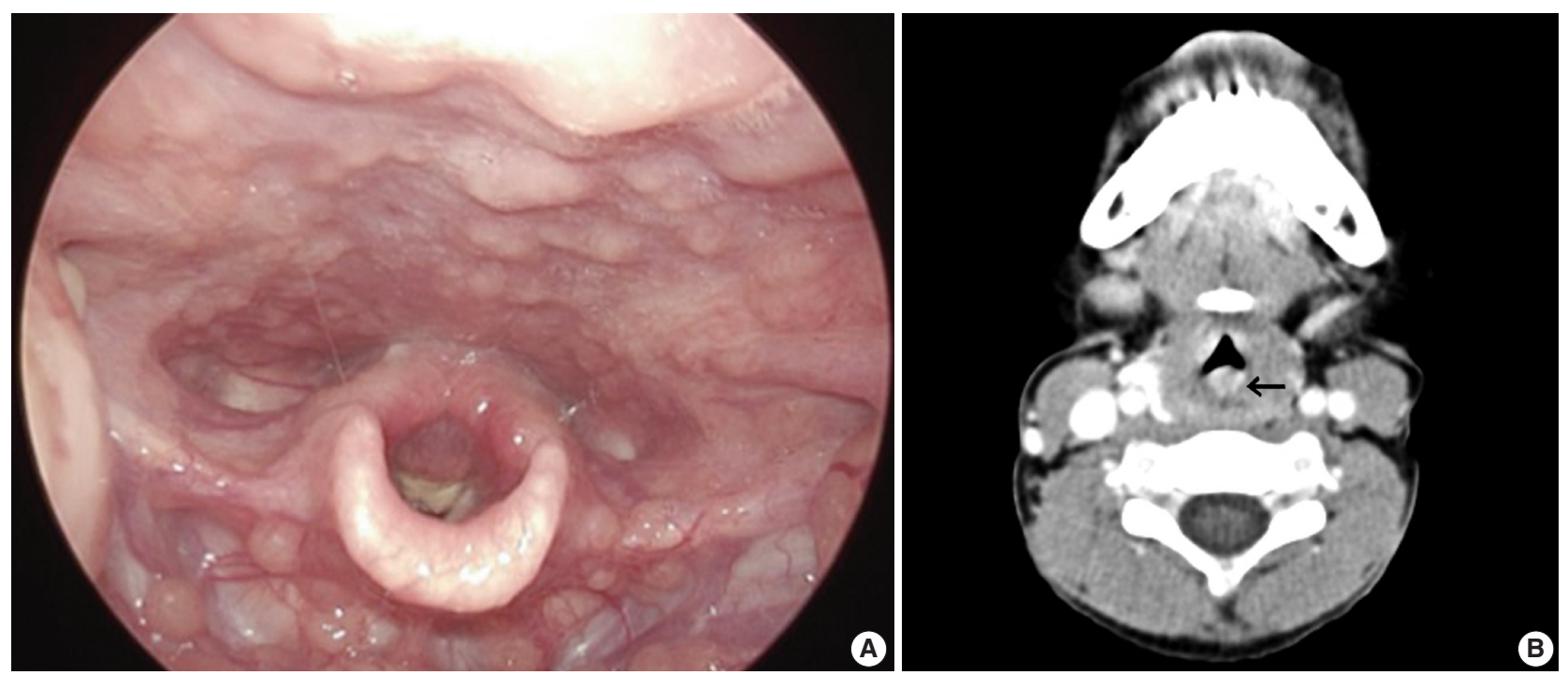

Fig. 1. (A) Laryngoscopy shows scattered swelling and a crust-like lump lesion, causing subglottic stenosis and stricture. (B) Head and neck computed tomography reveals a 2.2-cm-sized lesion (arrow) with heterogeneous enhancement in the posterior wall of the subglottic trachea.
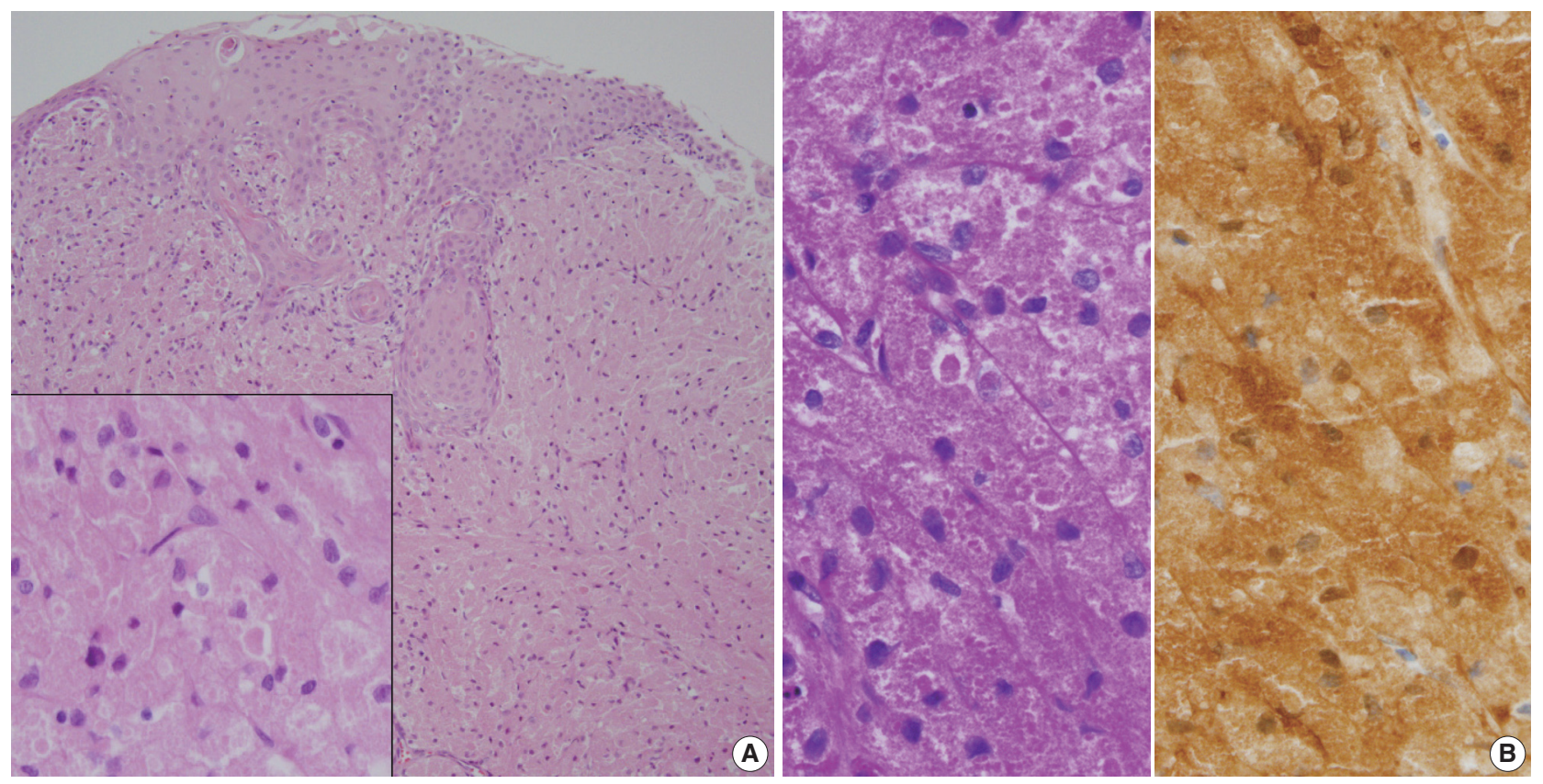

Fig. 2. (A) Large polygonal-shaped tumor cells fill the subepithelial stroma. Note the overlying pseudoepitheliomatous hyperplastic squamous epithelium. The inset indicates tumor cells containing fine granular cytoplasm. (B) Voluminous cells are strongly stained with periodic acid-Schiff with diastase-pretreatment (left) and S-100 protein (right).

positive for S-100 protein (1:600, polyclonal, Dako, Glostrup, Denmark; Fig. 2B, right) and negative for smooth muscle actin (1:100, 1A4, Dako). Because the tissue lacked cellular pleomorphism and mitosis, benign GCT was diagnosed.

Seven months later, the patient is healthy with remaining tumor. A 12-month follow-up visit is planned.

\section{DISCUSSION}

Pediatric laryngotracheal tumors are uncommon, accounting for only $2 \%$ of laryngeal lesions, and most of them are benign [2] . The most common occurrences are papillomatosis and hemangioma [3]. GCT most commonly affects the posterior portion of the larynx. Pediatric laryngeal GCT is rare [4], with fewer than 
20 cases of pediatric laryngotracheal GCTs reported [5]. In addition to its rare incidence, laryngotracheal GCT in children may present with stridor and upper airway obstruction, a very serious clinical presentation, and a long period of time may pass before exact diagnosis. Causes of airway distress vary from infection to rare neoplasm. Furthermore, a preoperative diagnosis of laryngotracheal GCT is not easy because tracheal tumors are infrequent in children. In children, ultrasound is favored over CT. Although preoperative diagnosis of laryngeal GCT may not be easy, its pathologic diagnosis through characteristic histology and immunohistochemical results can be assured, unless the biopsy sample is inadequate. If the diagnostic biopsy is purely superficial, only squamous epithelium will be examined, which may be mistaken as squamous cell carcinoma. Pathologic diagnosis of GCT is based on observation of its classic morphology: overlying pseudoepitheliomatous hyperplasia, submucosal polygonal or elongated cells with small nuclei, and eosinophilic granules stained by S-100 protein and PAS in cellular lysosomes.

Although most GCTs are benign, some show malignant behavior, producing controversial classification between benign, atypical, and malignant GCTs [6]. The pathological criteria of malignancy, i.e., the Fanburg-Smith criteria, have been the most useful. The histopathological diagnosis of malignancy is based on the following six criteria: necrosis, spindling, vesicular nuclei with prominent nucleoli, mitotic activity ( $>2$ mitoses $/ 10$ highpower fields under $200 \times$ magnification), high nuclear to cytoplasmic ratio, and pleomorphism. GCTs showing three or more of the criteria are categorized as malignant, those showing one or two criteria are categorized as atypical, and those showing only focal pleomorphism are categorized as benign.

If GCT occurs in multiple locations, it may be the result of multicentric origin rather than metastasis, as in the skin [7]. Multiple GCTs have been reported in the skin of children, and most of those cases were associated with various systemic syndromes, such as neurofibromatosis (NF), Noonan syndrome (NS), Costello syndrome, and mutation of the phosphatase and tensin homologue (PTEN) gene. The molecular pathogenesis of multiple GCTs has not yet been clarified. Alterations of the RAS/mitogen-activated protein kinase pathway promoting cellular proliferation and oncogenesis, i.e., gain-of-function mutations, may explain why NF1 and NS patients develop multiple occurrence of GCTs [7]. Therefore, long-term follow-up should be required in patients with pediatric laryngotracheal GCT.

Treatment of laryngotracheal GCT is poorly standardized due to limited data, particularly in children. Most pediatric GCTs take a benign course, unless the patient suffers airway obstructive symptoms, and there is no role for radiotherapy or chemotherapy [8]. Complete excision with a clear resection margin is regarded as the standard of treatment, though therapy may vary from simple observation to laryngotracheal reconstruction [9]. Endoscopic resection may be undertaken as the initial treatment for a small GCT. On the other hand, surgical resection is recommended for laryngotracheal GCTs measuring more than $8 \mathrm{~mm}$ or those with full thickness involvement of the airway for maintenance of the airway [10].

Following complete resection, recurrence of GCT is unlikely, and recurrence rates are around $2 \%-3 \%$ if it is completely excised. Recurrence and increased size in benign GCT may be indicators of malignant transformation. Pediatric patients with laryngotracheal GCT and incomplete conservative surgery should be closely and regularly followed to detect late recurrence due to incomplete resection, metachronous multiple occurrence, or systemic manifestation of an underlying syndrome as well as lifetime risk of recurrence.

In conclusion, pediatric GCT involving the trachea and larynx mimics papilloma or hemangioma and poses a challenging diagnosis. The present case was unique for two reasons: first, the laryngotracheal occurrence, an unusual location for GCT; and second, the presentation of acute stridor that necessitated a differential diagnosis that included entities with asthma or bronchitis. Although laryngotracheal GCT is rare, pediatricians and radiologists should bear it in mind when evaluating a patient presenting with respiratory obstructive symptoms.

\section{Ethics Statement}

The medical center's institutional review board approved this study with a waiver of informed consent (No. GFIRB2019-243), and the study was performed in accordance with the 1964 Helsinki Declaration and its later amendments.

\section{ORCID}

Jungsuk Ahn https://orcid.org/0000-0003-0312-2460

Na Rae Kim https://orcid.org/0000-0003-2793-6856

Yong Han Sun https://orcid.org/0000-0003-1527-6782

\section{Author Contributions}

Conceptualization: YHS. Data curation: JA. Investigation: JA, NRK. Resources: YHS. Supervision: NRK. Writing-original draft: NRK, JA. Writing-review and editing: NRK.

\section{Conflicts of Interest}

The authors declare that they have no potential conflicts of interest.

\section{Funding Statement}

No funding to declare. 


\section{References}

1. Dunaway CL, Brogdon BG, Robinson AE. Granular cell myoblastoma of the trachea. Pediatr Radiol 1981; 11:210-1.

2. Burton DM, Heffner DK, Patow CA. Granular cell tumors of the trachea. Laryngoscope 1992; 102: 807-13.

3. Holland RS, Abaza N, Balsara G, Lesser R. Granular cell tumor of the larynx in a six-year-old child: case report and review of the literature. Ear Nose Throat J 1998; 77: 652-60.

4. Amar YG, Nguyen LH, Manoukian JJ, Nguyen VH, O'Gorman A, Shapiro R. Granular cell tumor of the trachea in a child. Int J Pediatr Otorhinolaryngol 2002; 62: 75-80.

5. Spandow O, Lindholm CE. Granular cell tumour in a child's trachea: a diagnostic and therapeutic challenge. Int J Pediatr Otorhinolaryngol 1994; 30: 159-66.

6. Fanburg-Smith JC, Meis-Kindblom JM, Fante R, Kindblom LG.
Malignant granular cell tumor of soft tissue: diagnostic criteria and clinicopathologic correlation. Am J Surg Pathol 1998; 22: 779-94.

7. Schrader KA, Nelson TN, De Luca A, Huntsman DG, McGillivray BC. Multiple granular cell tumors are an associated feature of LEOPARD syndrome caused by mutation in PTPN11. Clin Genet 2009; 75: 185-9.

8. White JB, Glade R, Rossi CT, Bielamowicz S. Granular cell tumors of the larynx: diagnosis and management. J Voice 2009; 23: 516-7.

9. Pernas FG, Younis RT, Lehman DA, Robinson PG. Management of pediatric airway granular cell tumor: role of laryngotracheal reconstruction. Int J Pediatr Otorhinolaryngol 2006; 70: 957-63.

10. Daniel TM, Smith RH, Faunce HF, Sylvest VM. Transbronchoscopic versus surgical resection of tracheobronchial granular cell myoblastomas. Suggested approach based on follow-up of all treated cases. J Thorac Cardiovasc Surg 1980; 80: 898-903. 